\title{
L'OEUVRE OUVERTE DE TOMI UNGERER : TRADUCTIONS PALIMPSESTES ET TRANSFERTS INTERCULTURELS
}

\author{
Anne Schneider* \\ Conservatrice du Musée Tomi Ungerer \\ Thérèse Willer ${ }^{* *}$ \\ Conservatrice du Musée Tomi Ungerer
}

\begin{abstract}
Résumé: Tomi Ungerer, auteur et illustrateur pour enfants, né en France, à Strasbourg, en 1931 parle trois langues : le français, l'anglais, l'allemand, mais également le dialecte alsacien. Cette position linguistique particulière l'amène à superviser les traductions de ses albums. D'abord écrits en anglais, ses albums sont traduits en français et en allemand. De nombreux exemples montrent comment Tomi Ungerer joue avec les trois langues qu'il connaît bien, comment il crée des expressions, des jeux de mots, des sonorités qui proviennent de son imaginaire subversif. Au gré des traductions et retraductions, Tomi Ungerer rend son œuvre vivante,
\end{abstract}

\footnotetext{
* Agrégée de Lettres Modernes. Maître de conférences en langue et littérature françaises à l'Université de Caen et à l'ESPE (Ecole Supérieure du Professorat et de l'Education) depuis 2010. Spécialiste de littérature de jeunesse. Elle a enseigné pendant dix ans de la maternelle au lycée, puis comme agrégée de Lettres à l'IUFM de Strasbourg de 2001 à 2010. Elle est Vice-Présidente de l'Institut International Charles Perrault, à Eaubonne, centre de recherches en littérature de jeunesse. Eaubonne, France. E-mail: anne.schneider@unicaen.fr.

** Après des études de lettres classiques, d'archéologie et d'histoire de l'art à l'Université de Strasbourg. Docteur en histoire de l'art à l'Université de Strasbourg. Conservatrice en chef du patrimoine. Directrice du Musée Tomi Ungerer-Centre international de l'Illustration. Strasbourg, France. E-mail : therese.willer@strasbourg.eu.
} 
ouverte, créant un véritable palimpseste linguistique. Nous étudierons d'abord l'alsacianité dont fait preuve Tomi Ungerer, qui a passé son enfance en Alsace, puis nous parlerons des albums Les Trois Brigands et Otto qui ont été retraduits, enfin nous prendrons l'exemple de l'album Amis-Amies dont certaines expressions savoureuses ont été revues par l'auteur.

Mots-clés: Interculturalité. Traduction. Palimpseste. Linguistique

\title{
TOMI UNGERER'S PALIMPSESTIC WORK: A CASE OF INTERCULTURAL TRANSLATION AND INTERLINGUISTIC ADAPTATION
}

\begin{abstract}
Tomi Ungerer, author and illustrator of works for children, born in France, Strasbourg, in 1931, speaks three languages: French, English, German, and the Alsatian dialect. Therefore, his idiosyncratic linguistic fluency gives him an opportunity to supervise the translation of his pieces. Written primarily in English, his works are also provided - with his supervision - both in French and German. Many excerpts of these texts manifest how effectively Ungerer plays with all the languages he is acquainted with, as he creates expressions, puns, and sounds emerging from his subversive mind. If his concoction of these translations and retranslations are taken into consideration, it would be plausible to affirm that Ungerer has been setting forth a living and open work - a real linguistic palimpsest. This study focuses first on the Alsatian aspects present in Ungerer's work, whose childhood has been spent in Alsace. Afterwards, the focus shall be directed to two of his works - Les Trois Brigands and Otto - which have been retranslated; and, finally, the expressions which have been revised by the author, present in Amis-Amies are reflected upon. Keywords: Interculturality. Linguistics. Translation. Palimpsest.
\end{abstract}

Le monde de l'édition pour la jeunesse en France est marqué actuellement par l'anniversaire de la fondation, il y a cinquante ans, de la plus grande maison d'édition française pour enfants : L'Ecole des Loisirs.

Pour fêter cet événement, un partenariat commercial ${ }^{1}$ s'est $^{\text {'es }}$ conclu entre la marque bien connue de sous-vêtements pour enfants Petit-Bateau et L'Ecole des Loisirs. Le slogan commercial de la marque, « Les héros de L'Ecole des Loisirs sont chez Petit 
Bateau », s'appuie sur la création d'une collection intitulée « le bestiaire enchanté » mettant en scène sur des vêtements, tee-shirts, culottes, débardeurs, cinq héros de la maison d'édition pour les petits : le poussin de Claude Ponti, le loup de Mario Ramos, le crocodile de Dorothée de Montfreid, le chiot d'Iris de Moüy et le poulpe de Tomi Ungerer. Le site propose une biographie de chaque auteur, des extraits des albums et une interview de Louis Delas, actuel éditeur de la maison d'édition fondée en par Jean Fabre, Jean Delas et Arthur Hubschmid en 1965.

Quelle merveilleuse traduction que cette mise en scène du héros de Tomi Ungerer, héros éponyme de l'album Emile ${ }^{2}$, ce poulpe vert, fluide et malicieux aux qualités humaines et héroïques indéniables, naguère mis au ban du bestiaire traditionnel ! Car il s'agit là véritablement d'un phénomène connu en littérature de jeunesse : les héros des enfants poursuivent leur fortune littéraire par la novélisation, la médiatisation, les produits dérivés. Cette traduction ou ce transfert démontre non seulement, la notoriété de Tomi Ungerer et la place qu'il occupe au sein de L'Ecole des loisirs comme auteur-illustrateur phare, mais aussi la caractéristique de son œuvre, une oeuvre ouverte, qui est marquée par ses transferts, traductions, rééditions, retraductions qui en font une œuvre vivante, dynamique et créative.

Pour analyser ce concept «d'oeuvre ouverte »d' Umberto Ecco, conformément au titre de son ouvrage ${ }^{3}$, qui repense le rapport du lecteur à l'oeuvre, mais que nous entendons aussi du côté du rapport de l'auteur à sa propre œuvre, nous montrerons comment Tomi Ungerer traduit et retraduit ses œuvres dans une perspective ouverte, attentif à la fois à son public, enfant ou adulte, à la porosité des frontières linguistiques, à la dimension interculturelle qui lui est chère.

Nous montrerons d'abord comment le contexte linguistique et le concept d'alsacianité placent son oeuvre comme une œuvre linguistiquement ouverte, puis à travers l'exemple des albums Les Trois Brigands et Otto, nous analyserons les notions de traduction palimpseste $^{4}$, enfin, nous verrons comment la réédition de l'album AmiesAmis met en avant la figure d'un auteur-traducteur sans frontières. 


\section{I. « L'Alsacianité » : du régional à l'international}

\section{1) Un parcours linguistique complexe}

La biographie de Tomi Ungerer, indiquée sur le site de la marque Petit Bateau, se présente sous la forme d'un petit paragraphe :

« Tomi Ungerer, affichiste, auteur illustrateur, inventeur d`objets, collectionneur, dessinateur publicitaire, était un grand artiste à qui lıon doit d`inoubliables albums pour les enfants. Il a notamment reçu le prix Hans-Christian Andersen pour leensemble de son œuvre en 1998. »

Or, cette biographie néglige un détail important : l'ancrage de Tomi Ungerer en terre alsacienne. Comme le rappelle Thérèse Willer dans son article : "Tomi Ungerer l'alsacien ${ }^{5}$, cette dimension régionale participe de son identité. "Alsacien de naissance, Tomi Ungerer est devenu au cours de sa vie un « Weltbürger » qui s'est adapté tel un caméléon aux pays qu'il habite : «Partout où je vais je me sens tout de suite presque comme à la maison. J'entre immédiatement en contact avec les gens. Que ce soit en Irlande, au canada, ou comme autrefois à New-York. » En dépit de l'éloignement de sa région natale, l'artiste est resté Alsacien de coeur et ne cesse de se réclamer de cette identité. Son enfance et sa jeunesse en Alsace ont marqué profondément l'homme et l'oeuvre. » ${ }^{6}$

Tomi Ungerer, né en Alsace en 1931, a été élevé entre les deux guerres dans une famille bourgeoise, protestante et francophile. Lorsque l'Alsace fut occupée par les nazis, la pratique de la langue française se limita pour le jeune Tomi au cercle familial : « Le français était interdit par la loi, mais nous continuions en famille à le parler. $»^{7}$ De 1943 à 1944, il a aussi tenu tant bien que mal un journal en français, " aussi perfectionné que du petit nègre. ${ }^{8}$ 
Bien que cela ne fût pas non plus autorisé, il parlait le dialecte alsacien avec ses petits camarades. Puis, quand les Français libérèrent l'Alsace, des affiches proclamèrent le retour à la langue du pays : "C'est chic de parler français ${ }^{9}$. Ses nouveaux professeurs, des «Alsaciens de l'intérieur ${ }^{10}$ qui étaient partis pour la France libre, lui reprochèrent son accent alsacien. Il y avait de quoi en perdre son latin ! Est-ce pour cette raison que l'artiste ne se fixera dans aucune langue et que son parcours linguistique ressemble un peu à un melting-pot... ? Un rien provocateur, il lui est même arrivé d'adapter son accent selon le pays où il se trouvait, allemand en France, français en Allemagne, par exemple.

«Dans le "meilleur des mondes", je m'adaptais. Là se trouve l'origine de ce que j'ai appelé par la suite mon caméléonisme. Tout était remis en perspective par la complexité de la situation : Français à la maison, Allemand à l'école, Alsacien avec mes petits copains ", écrit-il dans $\grave{A}$ la guerre comme à la guerre ${ }^{11}$. Ceci explique qu'il soit capable de décentrement et de distanciation ; il réfléchit au statut de chacune de ces trois langues dans son imaginaire particulier et dans son parcours migratoire.

De l'aveu d'une de ses traductrices, Florence Seyvos, ${ }^{12}$ il supervise parfois les traductions (de l'anglais vers le français ou de l'anglais vers l'allemand), il y ajoute des éléments personnels, qui montrent qu'il se préoccupe de son lectorat enfantin et qu'il s'adresse à lui. Cette appétence particulière pour les langues - il a appris l'allemand en trois mois, l'alsacien dans la cour de l'école, l'anglais en deux ans - explique pourquoi il s'est soucié des traductions de ses œuvres, de leur passage de l'anglais, langue dans laquelle il écrit, vers le français ou l'allemand. Ungerer aime traduire et retraduire ses textes, en y ajoutant souvent sa patte personnelle, sensible au relief des mots, à leurs contours, aux expressions imagées que les expressions renvoient, mais aussi au contexte culturel, au peuple auquel il s'adresse. ${ }^{13}$ Traducteur et retraducteur au gré des éditions, il se positionne en linguiste qui passe alternativement d'une langue à l'autre, sans frontières, sans a priori, effaçant par là même les rapports de domination qui peuvent s'exercer entre elles. 
Le 4 décembre 2013, lors de l'émission sur France Inter « Le grand entretien » animée par François Busnel, Tomi Ungerer expliqua son rapport avec les langues : le français, l'alsacien, l'allemand, l'anglais. Il explique que « les langues n'ont pas de passage à niveau ", " les mots sont là en file d'attente et attendent d'être employés. $\gg^{14}$

Un témoin et ami de la famille Ungerer rapporte que pendant les études du jeune homme à Strasbourg au début des années 1950, de mystérieuses disputes éclataient entre lui et sa mère au sujet de la pratique de l'allemand et de l'alsacien. ${ }^{15}$ Lui a-t-il reproché d'avoir parlé exclusivement le français avec ses enfants ? Alice Ungerer considérait-elle l'alsacien comme une langue inélégante, indigne du milieu bourgeois dont elle était issue ou était-ce une preuve de sa francophilie?

C'est en tout cas avec l'anglais, appris à l'école, que son fils se familiarisa en fréquentant le centre culturel américain, dans le but avoué de partir pour New York. D'une certaine manière, cette langue symbolisait pour lui la liberté car elle lui permettait d'échapper aux contraintes linguistiques qu'on lui avait imposées dans sa jeunesse et de commencer une nouvelle vie. Débarqué aux ÉtatsUnis en 1956, puis installé définitivement à New York en 1957, le jeune artiste fut confronté avec la langue du pays d'autant plus rapidement qu'il dut s'insérer dans la vie professionnelle. L'un de ses premiers contacts fut Ursula Nordstrom, directrice du département jeunesse des éditions Harper \& Brothers à New York. ${ }^{16}$ Dès 1956, il lui présenta des manuscrits et des projets de livres pour enfants dont les textes étaient en anglais.

Quatre histoires de la famille Mellops ont été éditées entre 1957 et 1963 chez Harper \& Brothers, devenu plus tard Harper \& Row, qui ont connu un succès immédiat aux États-Unis. D'après les archives de la maison d'édition, ${ }^{17}$ on sait que les manuscrits faisaient l'objet de multiples corrections pour être adaptés à la mentalité américaine, Tomi Ungerer étant considéré comme un auteur à l'esprit trop français. Au-delà de l'obligation évidente de le pratiquer, l'anglais est devenu la langue de prédilection de l'auteur, tant à 
l'oral qu'à l'écrit. Au cours des années 1960, Tomi Ungerer se sentait tellement à l'aise qu'il inventa un slogan pour une série d'affiches destinée initialement à promouvoir la loterie new-yorkaise, mais qui servit finalement à la publicité du magazine The Village Voice : " Expect the unexpected ", «Attendez-vous à l'inattendu », une expression passée par la suite dans le langage courant et dont Tomi Ungerer se montre aujourd'hui encore très fier ${ }^{18}$.

Après les années passées à New York, l'artiste s'est fixé en 1971 en Nouvelle-Écosse, au Canada, puis à partir de 1976 en Irlande. Son environnement familial et professionnel continuait d'être anglophone. Burton Pike, professeur de littérature comparée et ami new-yorkais de longue date de l'artiste, s'est occupé en 1973 des corrections du texte que Tomi Ungerer avait écrit en anglais pour le livre Far Out Isn't Far Enough (cf. illustrations ci-jointes). ${ }^{19}$ Ce reportage illustré sur sa vie en Nouvelle-Écosse a été réédité en France sous le titre Nos années de boucherie. Dans son témoignage, Burton Pike raconte que les corrections qu'il y a apportées n'avaient rien de compliqué, car l'anglais de l'auteur était excellent. ${ }^{20} \mathrm{Il}$ a conservé ses constructions de phrases car ses exagérations et ses tournures étaient délibérées et démontraient une réelle originalité linguistique. Il cite l'exemple du terme de septic tank (fosse septique) : Tomi Ungerer a écrit l'adjectif sceptic avec la lettre «c ", comme dans le mot homonyme "sceptique ", jouant du décalage entre les deux termes. Il a aussi usé de néologismes comme le mot funerated qui n'existe pas en anglais, dans la phrase "We funerated the departure of our cow $»^{21}$ : "Nous avons célébré les funérailles de notre vache ». Le titre du livre est également de son cru. À Far out, une expression populaire qui signifie "dernier cri », Tomi Ungerer a ajouté, Isn't far enough, en créant ainsi un jeu de mots : «La Nouvelle-Écosse est bien loin, mais pas assez loin ». Et Burton Pike d'ajouter que la traduction allemande du titre Heute hier, morgen fort (Aujourd'hui ici, demain ailleurs) est tout à fait adaptée. La traduction française, en revanche, n'a absolument pas tenu compte de cette expression et a choisi de mettre l'accent sur l'une des activités principales de l'artiste dans sa ferme 
canadienne, tuer le cochon, ce qui rend le titre à peu près incompréhensible pour le néophyte.

Tout donne donc à penser que Tomi Ungerer a trouvé avec l'anglais une langue qui lui convenait, notamment parce qu'elle est pragmatique. Il lui semble qu'elle exprime mieux sa pensée que ne sauraient le faire le français et l'allemand, deux langues qui lui sont pourtant bien plus proches : "Une langue qu'il s'est appropriée et qu'il a intériorisée au point qu'elle coule de lui comme un torrent étincelant », précise Burton Pike dans un essai à propos du reportage de l'artiste sur ses années canadiennes. ${ }^{22}$ Pendant toute la période new-yorkaise de Tomi Ungerer, la publication de ses livres obéissait à un rituel immuable : ils étaient d'abord édités aux États-Unis, puis en Europe, par Diogenes Verlag en allemand et par l'École des loisirs en français. ${ }^{23}$ Tous les manuscrits étaient rédigés en anglais, comme par exemple celui de The Hat, devenu par la suite Der Hut ${ }^{24}$ et Le Chapeau volant ${ }^{25}$ (cf. illustrations ci-jointes). La dernière édition originale d'un livre pour enfants parue aux États-Unis fut celle, en 1974, de The Storybook of Tomi Ungerer (Le livre des contes de Tomi Ungerer). Allumette, son dernier livre pour la jeunesse, avant une pause de près de vingt ans, a été édité la même année chez Diogenes Verlag à Zurich ${ }^{26}$. À partir de cette date, tous les ouvrages de Tomi Ungerer paraîtront d'abord en langue allemande chez l'éditeur suisse. Même si l'usage de l'anglais ne constituait donc plus une obligation pour l'auteur, il a cependant perduré sans exception dans ses textes.

Interrogé en 2004 au sujet de sa collaboration éditoriale de longue date avec Tomi Ungerer, ${ }^{27}$ Daniel Keel, le fondateur de Diogenes $\operatorname{Verla}^{28}$, raconte que lorsque Tomi Ungerer avait un projet à lui proposer, ils en discutaient et faisaient le layout ${ }^{29} \mathrm{en}-$ semble. L'auteur écrivait en anglais, et le texte était ensuite traduit en allemand par Anna Kramer-Clett, qui a fidèlement suivi tous les livres pour enfants de l'auteur. Certains choix de traduction en allemand ont posé des problèmes à Mme von Cramer-Klett, contraignant l'éditeur à prendre des options. En principe, l'auteur devait valider les traductions de ses ouvrages pour éviter qu'on ne 
trahisse sa pensée. $\mathrm{Si}$, pour certains albums, il s'est peu impliqué, pour d'autres, il a parfois modifié la traduction, s'auto-retraduisant lui-même.

\section{2) L'écriture palimpseste d'une ouvre ouverte}

On peut donc considérer que ce bouillonnement linguistique originel propre à Ungerer a abouti parfois à de véritables réécritures de son œuvre, à des transferts, des retraductions ou des modernisations dans la mesure où l'écriture est considérée par Ungerer comme le lieu d'un jeu linguistique permanent. Ainsi, du melting-pot originel constitutif de sa pensée et de son cheminement identitaire au palimpseste créatif, la démarche est quasiment la même : l'appropriation de la langue procède par accumulation, ajouts, suppressions, insertions. Sans reprendre la terminologie de Gérard Genette qui considère la notion de palimpseste au sens de «transtextualité », nous préférons utiliser ce terme au sens de retouches, de réécritures. Ce qui est important pour lui c'est de toucher au plus près, à la fois l'âme et les sensations enfantines, de garder ses textes vivants et de leur donner un souffle, une voix, ce qu'il réussit parfaitement. En effet, nous pourrons avancer dans son œuvre trois types de palimpsestes linguistiques : un palimpseste euphonique, fondé sur des changements phonétiques nécessaires au rendu quasi physique de la langue -le fameux accent présent chez certains de ses personnages ; un palimpseste lexical reposant sur quelques mots choisis auquel il va conférer une véritable épaisseur, enfin, un palimpseste narratif auquel il sera attentif du point de vue de la logique de l'histoire- il s'adresse à des enfants donc il est dans une écriture réellement intentionnelle. Ainsi, les exemples que nous allons développer relèvent parfois de la retraduction, lorsque les réécritures concernent la modernisation de l'œuvre et sa republication, donc sa réception contemporaine, mais aussi de palimpsestes, au sens de réécritures ponctuelles sur le manuscrit ou d'ajouts, de suppressions, d'insertions ; cette démarche nous sem- 
blant révélatrice de l'attention permanente d'Ungerer à la langue et à sa traduction identitaire.

\section{II- Les Trois brigands et Otto, des œuvres ouvertes aux tra- ductions/retraductions multiples}

\section{1) Tomi Ungerer ou le palimpseste sonore des langues}

Tomi Ungerer écrit presque tous ses textes en anglais. Certains textes sont retouchés par lui, d'autres non, bien qu'il relise toutes les traductions, étant tout à fait à même de comprendre les versions française et allemande. Car Ungerer habite ces trois langues de façon intense ; elles lui sont tour à tour réservoirs d'images, de rythmes et de sons. Derrière ce triptyque bien identifié se situe pourtant l'alsacien, signe du plurilinguisme littéraire de l'auteur. En effet, chacune de ses langues est également mâtinée de l'alsacien, " [1]'autre langue à portée de voix », pour reprendre le titre de l'essai d'Yves Bonnefoy. ${ }^{30}$ Cet ancrage se retrouve dans l'utilisation de la «triphonie », qui confine à « l'europhonie », théorisée par l'écrivain alsacien Adrien Finck ${ }^{31}$. En effet, celui-ci, qui partage sa réflexion sur l'identité alsacienne avec d'autres auteurs alsaciens comme Tomi Ungerer, Claude Vigée ${ }^{32}$ ou André Weckmann $^{33}$, avance l'idée que le signifié engage le signifiant et que la dimension sonore et rythmique de la langue est très importante. Ainsi, les réécritures palimpsestes d'Ungerer montrent à quel point il aime les langues jusque dans leurs profondeurs sonores.

L'exemple le plus frappant réside dans la conversation téléphonique entre Oscar et David dans Otto ${ }^{34}$, alors qu'ils se retrouvent enfin des années plus tard. Le contexte émotionnel est très fort : deux amis allemands, David et Oskar, que la guerre 39-45 a séparés se retrouvent quarante ans plus tard aux Etats-Unis grâce à l'ours en peluche Otto, qu'ils possédaient à l'époque. Voici leur conversation téléphonique : "Allô ? Qui ? Quoi ? Z'est imbo- 
zible... Toi, David, tu es dans zette ville... Oui, Otto est là afec moi, oui... J'ariffe tout de zuite, donne l'adresse... " $^{35}$

De l'aveu de la traductrice de l'Ecole des Loisirs, Florence Seyvos, Ungerer a rajouté des éléments linguistiques sonores pour rendre au plus près l'oralité de la conversation. Ce palimpseste euphonique a pour effet de produire une conversation dont la dimension sonore permet de replonger immédiatement dans le contexte de la guerre, de l'Allemagne et d'opérer une sorte de reconnaissance auditive entre les personnages.

Cet exemple de «triphonie » montre à quel point la langue est le véhicule d'un imaginaire musical particulier, mais aussi d'une distanciation humoristique : Ungerer se situe dans un « entre-deux/ trois » linguistique, le français, l'allemand, l'alsacien.

D'autres exemples montrent à quel point celui-ci sait exactement ce qu'il veut et comment il intervient pour défendre ses idées. Ainsi, pour le nom de l'ours, «Otto », que la traductrice allemande Anna von Cramer-Klett voulait traduire par «Veilchen », ce qui signifie "violette " à cause de l'œil au beurre noir porté par l'ourson (en allemand, on parle de Veilchen pour dire « œil au beurre noir»), cette idée ne plaisait pas du tout à Ungerer, qui l'a immédiatement écartée. Elle note qu'elle fait des propositions qui sont validées ou non par lui. Dans le cadre de cet exemple, on peut garder l'idée qu'il a un imaginaire précis de ce qu'il souhaite et que le nom d'Otto lui paraît suffisamment universel, affectif et sonore pour être gardé dans toutes les langues, à plus forte raison en allemand, car c'est un prénom allemand. Il s'agit donc à la fois d'un palimpseste euphonique et lexical. C'est donc par ces exemples que nous comprenons comment la langue est vivante pour Ungerer et comment ces réécritures, rajouts, suppressions constituent pour lui une incarnation sonore de la langue, mais aussi une prise en compte de son destinataire enfantin pour lequel il veut se mettre à la portée. 


\section{2) Les réécritures des Trois brigands au gré du circuit éditorial}

Par exemple, l'album Les Trois brigands a été écrit en anglais, puis traduit en allemand puis en français et à nouveau remanié par Ungerer pour offrir une nouvelle version anglaise. Il offre un circuit éditorial particulier, qui montre qu'il s'agit là d'une œuvre vivante et que ses diverses traductions et retraductions lui donnent à chaque fois une nouvelle vie, l'enrichissent et contribuent à sa longévité et à sa prospérité. Voyons donc ces passages d'une langue à l'autre.

C'est en 1961 à Munich que naît la première édition Die drei Räuber, publiée par Georg Lentz. La version originale en anglais a été proposée à Ursula Nordstrom, des éditions Harper \& Row, et refusée par elle : les couleurs, le texte, la petite fille enlevée par les brigands, tout cela ne lui plaisait guère. Cependant, en 1962, les éditions américaines Atheneum publient le texte sous l'appellation The Three Robbers, mais ils suppriment la morale d'origine : "Quelle que soit la couleur de l'argent, il n'est jamais trop tard pour en faire un bon usage ${ }^{36}{ }^{\prime}$, que l'on ne retrouvera plus par la suite dans aucune version du texte d'Ungerer quelle que soit sa langue. Cette omission affadit nettement le texte et, à cette morale très ungerérienne, plutôt subversive, se substitue une conclusion bien sage : « À la fin, ils bâtirent une muraille tout autour de la ville avec trois tours imposantes, une pour chaque brigand, pour les remercier. ${ }^{37}$ Troisième étape de ce circuit, en 1963, la récupération du texte allemand par Daniel Keel, après le rachat de la maison Georg Lentz, permet sa republication sous le titre Die Drei Räuber chez Diogenes Verlag. En 1968, l'École des Loisirs publie la version française des Trois brigands, traduite de l'allemand par Tilde Michels. Le 17 janvier 2008, une réunion réunit au Musée Tomi Ungerer la conservatrice du Musée Ungerer, Tomi Ungerer et des représentants des éditions Phaidon Presse avec pour objectif une réédition des Trois brigands en anglais et pour une modernisation de celle-ci. ${ }^{38}$ 
Cette nouvelle version paraîtra en mai 2009, les modifications portant à la fois sur le texte et sur l'image. La retraduction concerne ici l'image, ce qui est assez rare et plutôt original. Pour celle-ci, ce sont surtout des raisons économiques qui conditionnent les modifications : typographie plus moderne, couleurs des pages, écriture sur un fond bleu au lieu du noir ; Ungerer ajoute même le dessin d'un troisième brigand dans la page finale, ce qui créé un effet de symétrie. En effet, à l'origine, on ne trouvait que deux brigands dans l'image. C'est donc un palimpseste narratif dans la mesure où le traitement de l'image relève de la narration au même titre que l'écrit. Quant au texte, quelques phrases sont modifiées par Tomi Ungerer entre la version publiée chez Atheneum et celle de chez Phaidon, en s'appuyant également sur la version de chez Diogenes - par exemple, la phrase "So kow you's der Dreier » est remplacée par "fratherd all the lost ", ou encore la phrase : "So they set off and gathered up all the lost, unhappy and abandoned children they could find " remplace la phrase allemande de chez Diogenes : "[Und weil Tiffany, das Waisenkind, ihnen so gut gefiel], gingen sie auf die Suche nach anderen unglücklichen und verlassenen Kindern, um für sie zu sorgen " (sur la double page de la colonne de carrioles transportant des enfants). Ces changements sont le fait $\mathrm{d}>$ Ungerer qui a émis ces propositions.

Si les modifications sont somme toute minimes, elles montrent à quel point le texte est vivant et combien il peut sortir des oubliettes et retrouver une seconde jeunesse. Ainsi, à la suite d'un grand article publié dans le New York Times offrant une rétrospective de l'œuvre de Tomi Ungerer, Brad Bernstein, se remémorant la grande époque de Tomi Ungerer dans les années 1960 aux ÉtatsUnis, décide alors de faire connaître à nouveau l'œuvre de Tomi Ungerer, de la dépoussiérer. Ainsi, il fut à l>origine de la nouvelle version du dessin animé « Jean de la Lune » qui naîtra de cette initiative. Dans tous les cas, Ungerer rajoute sa patte, que ce soit par sa propre voix off dans le film Les Trois Brigands ou Jean de la Lune ou par ses ajouts et ajustements afin de moderniser ses textes. 


\section{La réédition d'Amis-amies : auto-traductions et retra- ductions}

Une séance de travail réunissant Tomi Ungerer et l'équipe éditoriale de Phaidon Press le 18 janvier 2008 au Musée Tomi Ungerer a pu être suivie par l'une des auteures de cet article. ${ }^{39}$ Après avoir réédité Les Trois brigands et divers autres livres pour enfants de Tomi Ungerer, Phaidon avait mis à l'ordre du jour la réédition du livre paru en 2007 chez Diogenes Verlag sous le titre Neue Freun$d e$, et à l'École des loisirs sous le titre Amis-amies. En feuilletant les deux versions existantes, Tomi Ungerer a d'abord relevé une interversion des images entre la page 32 et la page 33, par rapport à la maquette initiale : il s'agissait d'une erreur de mise en page de départ. Il a souhaité superviser les traductions pour la réédition par Phaidon Press. Les textes qu'il avait écrits à l'origine allaient donc être comparés avec ceux qui seraient proposés par la maison d'édition. Mais, lors de la séance de travail, seuls les livres français et allemand furent mis en parallèle, sans retour sur le manuscrit original en anglais parce qu'il était introuvable. La réédition devait comporter deux versions, l'une pour les États-Unis, l'autre pour l'Angleterre ${ }^{40}$. Tomi Ungerer tenait à une grande précision dans la terminologie. Par exemple, (16), pour la traduction du mot " râler ${ }^{41}$ qui reprenait le terme allemand "schimpfen ", a été choisi le terme anglais de «groaned», qui semblait le plus adaptét2. La phrase en français "ça fera une exposition fantastique » (26), qui reprenait directement celle en allemand "Das wird eine tolle Ausstellung », devait être traduite par «It will be a real blockbuster» («ce sera une vraie superproduction»). Certains jeux de mots et expressions se sont révélés particulièrement délicats à traduire, comme, par exemple, «Les poubelles au pouvoir» ou « Einfall statt Abfall ! (25), et qui allait être remplacé par «Art runs amok» ${ }^{43}$. Il n'a pas été possible de vérifier le résultat de cette séance de travail, le livre Amis-amies n'ayant pour l'instant pas encore fait l'objet de la réédition prévue chez Phaidon Press. 


\section{La/les langue/s de Tomi Ungerer, intraduisible/s ?}

Tomi Ungerer saute allègrement d'une langue à l'autre, les mêle avec jubilation, à la manière de son prédécesseur, le poète et artiste dada Jean-Hans Arp, né lui aussi en Alsace, et qui, lui aussi, associait le français, l'allemand et l'alsacien. Pour Jean-Claude Walter, "Arp part d'un mot, il en découvre et fait jaillir le sens - dans tous les sens, et toutes les directions - comme en un rêve, créant sans cesse des images, qui, à leur tour... $»^{44}$ Les calembours, expressions de l'humour germanique tirant son nom d'un aristocrate allemand qui l'aurait inventée, ainsi que les jeux de mots et les termes imagés, sont habituels à Tomi Ungerer. Il les pratique dans toutes les langues qu'il connaît, en les enchevêtrant. C'est de cette manière qu'il a imaginé par exemple les légendes satiriques qui accompagnent la description graphique d'une soirée mondaine dans les années 1960 à New York, pour le livre The Party : entre autres nombreux exemples, le nom d'une richissime américaine, Mrs Julia van Flooze, a été imaginé à partir d'un jeu de mots autour de " flouze », qui désigne l'argent en argot. ${ }^{45}$ La partition est à cet égard particulièrement difficile pour un traducteur ... et pour un correcteur : combien s'y sont trompés en voulant à tout prix corriger ce qu'ils ont pris pour des fautes d'orthographe et de syntaxe de l'auteur et qui n'étaient que des néologismes et de pures créations.

Les propos de Jean-Claude Walter à propos de la poétique de Arp : " À partir des jeux avec les mots, Arp va découvrir "sa" langue - cette métaphore de la vie intérieure qu'il évoque au sujet de ses dessins $»^{46}$, s'appliquent à Tomi Ungerer qui, à son tour, a inventé la sienne, pleine de fantaisie et d'humour. Elle est fondée sur le melting-pot, véritable art de vivre, mais aussi sur un palimpseste originel traumatique, transformé en résilience ${ }^{47}$, comme le montre cette anecdote vécue dans son école à la Libération. Il avait enveloppé son index droit d'une bande de sparadrap pour éviter d'être puni de ne pas avoir fait son devoir de français : «Ce pansement, devenu symbolique, je l'ai arraché. Ma langue ? Elle avait déjà été arrachée. Heureusement qu'à cet âge, sans greffe et 
sans anesthésie, la panoplie des organes repousse tout comme la queue des lézards. ${ }^{48}$

Voilà pourquoi, l'œuvre de Tomi Ungerer témoigne d'un palimpseste linguistique permanent. Sa langue intérieure, croisement de plusieurs voix, fait de son œuvre " une œuvre ouverte », pour reprendre dont les significations sont à décoder et pour lesquels la place du lecteur-adulte et du lecteur enfant est sans cesse à reconsidérer.

\section{Notes}

1. http://www.petit-bateau.fr/e-shop/Landing/lp-ecole-des-loisirs-1/1/le-partenariat.html, consulté le 12/06/2015

2. Tomi Ungerer, Emile, L'Ecole des Loisirs, 1978.

3. L'œuvre ouverte, Umberto Ecco, Seuil, Points, 1965.

4. Selon Gérard Genette, Palimpsestes, Editions du Seuil, 1982

5. Thérèse Willer « Tomi Ungerer l'alsacien » Saisons d'Alsace, Hors série, avril 2015, $22-31$

6. Thérèse Willer, op. cit., 22

7. À la guerre comme à la guerre, op. cit. , 53.

8. Ibid. 
9. Expression en usage après la seconde guerre mondiale en Alsace.

10. Expression consacrée en Alsace pour désigner les non-alsaciens du reste de la France.

11. Tomi Ungerer, À la guerre comme à la guerre, dessins et souvenirs d'enfance, Édition de la Nuée Bleue, 1991, p. 50

12. Florence Seyvos, entretien du 20/04/2011.

13. L'article de Britta Benert et Christine Hélot traite de cette auto-traduction et de la question de la réception : "Tomi Ungerer, Homo viator, trois langues et quatre récits pour penser la notion d'identité », Revue Romanistik und Gesellschaft, Grenzgänge 14 (2007), H. 27, S. 167-168.

14. Émission « Le grand entretien » animée par François Busnel, France Inter, 04/12/2013, 17 h, invité : Tomi Ungerer.

15. Archives personnelles, TW.

16. Cf. à ce sujet TW, Tomi Ungerer. Graphic Art, Paris, Éditions du Rocher, 2011.

17. Archives personnelles, TW.

18. Stéphane Muller, Tomi Ungerer, Un point c'est tout, Paris, Bayard, 2011, 69

19. Londres, Methuen, 1984. Archives personnelles, TW.

20. Archives personnelles, TW.

21. Far Out Isn't Far Enough, manuscrit inédit, coll. privée, n. p.

22. Catalogue d'exposition Tomi Ungerer. Les années canadiennes : Here Today, Gone Tomorrow, Éditions Les Musées de la Ville de Strasbourg, 2010, 20. 
23. À l'exception du livre Les Trois brigands, qui parut d'abord en Allemagne sous le titre Die drei Räuber.

24. Zurich, Diogenes Verlag, 1972

25. Paris, l'Ecole des Loisirs, 1971

26. 1974

27. Archives personnelles, TW.

28. Grande maison d'édition suisse qui s'était spécialisée dans les arts graphiques dès les années 1950

29. Terme utilisé par Daniel Keel dans l'entretien, correspondant à " mise en page » en français

30. Yves Bonnefoy, L'Autre langue à portée de voix : essai sur la traduction de la poésie, Paris, Seuil, « Librairie du XX Ième siècle », 2013.

31. Voir notamment ses travaux de poète alsacien, mais aussi d'universitaire : Adrien Finck, La Stratégie du lierre : essai sur l'identité alsacienne, Le Drapier, Strasbourg, 1994 et Adrien Finck, L'allemand en Alsace / Die Deutsche Sprache im Elsass, actes du colloque de Strasbourg des 28-30 novembre 1985, réunis par Adrien Finck et Marthe Philipp, Presses universitaires de Strasbourg, 1988.

32. Ecrivain alsacien d'origine juive qui a beaucoup écrit sur le mixte tolérant des cultures en Alsace, en particulier dans ses romans Un panier de houblon, Tome 1 et 2, JC Lattès, 1994 et 1995

33. Poète et romancier alsacien qui a écrit sur Les Nuits de Fastov, Colmar, Alsatia, 1968, décrivant la dure condition des Malgrés-nous et, entre autres, Fonse ou l'éducation alsacienne, Paris, Oswald, 1975.

34. Voir à ce propos notre article : Anne Schneider, "Écarts de traduction et interculturalité : Otto de Tomi Ungerer, versions anglaise, allemande et françai- 
se”, in Virginie Douglas (dir), État des lieux de la traduction pour la jeunesse, PURH, 2015.

35. Tomi Ungerer, Otto, op.cit., 31.

36. Cf. à ce sujet Michael Patrick Hearn, "S'attendre à l'inattendu avec Tomi Ungerer et ses livres pour enfants ", Actes du colloque Tomi Ungerer. Les années new-yorkaises, Strasbourg, Edition Association T.C/70 Ans de Tomi Ungerer/ Musées de Strasbourg, 2002, 10 -35.

37. Ungerer, Les Trois Brigands, L’Ecole des Loisirs, 1968, excipit.

38. Archives personnelles, TW

39. Archives personnelles, TW

40. Les différences étaient de l'ordre du vocabulaire

41. «Regardez donc ces étrangers, râla un passant...»

42. Tomi Ungerer préférait ce terme

43. Les traductions sont dans ce cas précis, de l'auteur

44. Jean-Claude Walter, "Notes sur la poétique de Arp ", "Jean Hans Arp », Saisons d'Alsace, $\mathrm{n}^{\circ} 93$ (sept.1986), 85 .

45. The Party, New York, Grosmann Publishers, Paragraphic Books, 1966, non paginé. Le dessin original ne comporte pas la légende qui figure dans le livre.

46. « Jean Hans Arp », Saisons d'Alsace, nº93 (sept.1986), 85

47. Référence à la théorisation de Boris Cyrulnik qui parle ainsi de la capacité du sujet à surmonter un grand traumatisme. Voir entre autres, Boris Cyrulnik et Claude Seron (dir.), La Résilience ou Comment renaître de sa souffrance, éd. 
Fabert, coll. « Penser le monde de l'enfant », Paris, 2004

48. À la guerre comme à la guerre, op. cit. , 118.

\section{Bibliographie Corpus}

Ungerer, Tomi. The Party. New York: Grosmann Publishers, Paragraphic Books, 1966

Ungerer, Tomi. Far Out Isn't Far Enough. Londres: Methuen, 1984

Ungerer, Tomi. Les Trois Brigands. Ecole des Loisirs, 1968

Ungerer, Tomi. Otto. Ecole des Loisirs, 1999

Ungerer, Tomi. A la guerre comme à la guerre, dessins et souvenirs d'enfance. Strasbourg : Edition de la Nuée Bleue/DNA, 1991

Ungerer, Tomi. Amis-amies. Ecole des Loisirs, 2007

\section{Bibliogaphie critique}

Benert, Britta; Hélot, Christine. «Tomi Ungerer, Homo viator, trois langues et quatre récits pour penser la notion d'identité.» Romanistik und Gesellschaft, Grenzgänge, 14 (2007), H. 27, S. 167-168. 
Bonnefoy, Yves. L'Autre langue à portée de voix : essai sur la traduction de la poésie. Paris, Seuil : Librairie du XX I'me siècle, 2013.

Busnel, François; Ungerer, Tomi. Émission radiophonique: « Le grand entretien » animée par François Busnel. France Inter, 04/12/2013, 17 h, invité: Tomi Ungerer.

Cyrulnik, Boris ; Seron, Claude. La Résilience ou Comment renaître de sa souffrance. Fabert, coll., Penser le monde de l'enfant, Paris, 2004

Ecco, Umberto. L'œuvre ouverte. Seuil : Points, 1965.

Finck, Adrien. La Stratégie du lierre: essai sur l'identité alsacienne. Le Drapier : Strasbourg, 1994

Finck, Adrien ; Philipp, Marthe. L'allemand en Alsace / Die Deutsche Sprache im Elsass. Actes du colloque de Strasbourg des 28-30 novembre 1985, Presses Universitaires de Strasbourg, 1988.

Genette, Gérard. Palimpsestes. Editions du Seuil, 1982

Patrick Hearn, Michael. "S'attendre à l'inattendu avec Tomi Ungerer et ses livres pour enfants.» Actes du colloque Tomi Ungerer. Les années new-yorkaises, Strasbourg : Edition Association T.C/70 Ans de Tomi Ungerer/Musées de Strasbourg, 2002, $10-35$

Schneider, Anne. «Écarts de traduction et interculturalité: Otto de Tomi Ungerer, versions anglaise, allemande et française". Virginie Douglas, État des lieux de la traduction pour la jeunesse, PURH, 2015.

Vigée, Claude. Un panier de houblon. Tome 1 et 2, JC Lattès, 1994 et 1995

Walter, Jean-Claude. " Notes sur la poétique de Arp ». Jean Hans Arp, Saisons d'Alsace, $\mathrm{n}^{\circ} 93$ (sept.1986), 85 
Willer, Thérèse. Tomi Ungerer. Les années canadiennes: Here Today, Gone Tomorrow. Catalogue d'exposition Musée Tomi Ungerer, Strasbourg : Editions Les Musées de la Ville de Strasbourg, 2010

Willer, Thérèse. Tomi Ungerer. Graphic Art. Paris : Editions du Rocher, 2011

Weckmann, André. Les Nuits de Fastov. Colmar : Alsatia, 1968

Weckmann, André. Fonse ou l'éducation alsacienne. Paris : Oswald, 1975.

Recebido em: 28/07/2015 Aceito em: 15/09/2015 